\title{
Direct Calculation of Natural Orbitals of Two-Electron Systems
}

\author{
Heinz Kleindienst and Kai Rossen \\ Institut für Physikalische Chemie, Lehrstuhl I, Universität Düsseldorf
}

Z. Naturforsch. 40 a, 995-997 (1985); received March 23, 1985

A new method is proposed, which allows for the determination of the ground state energy and the natural orbitals (NO's) of a two-electron system directly and simultaneously. The basis for this calculation is a system of integrodifferential-equations, which defines those NO's.

\section{Introduction}

Natural orbitals of two-electron systems are of new interest since they have been used successfully in the PNO-CI-procedure [1]. As is wellknown, the definition and the first evaluation of the NO's goes back to Löwdin [2]. Thereafter several indirect and direct methods, i.e. methods with or without knowledge of the wavefunction, for the calculation of the NO's have been given, cf. [3], [4], [5], [6] and others.

In this paper a new method is proposed, which allows to calculate the NO's immediately from the system of integrodifferential equations defining the NO's. The basic idea is the use of a gradient method to minimize a certain quadratic functional in a Hilbertspace. It is shown that the solution of this "Extremal problem" is equivalent to the determination of the NO's.

Remark: The method was developed to determine natural nuclear and electronic orbitals ("non-BornOppenheimer orbitals") directly; this will be shown in a subsequent paper. Such orbitals were proposed by Bishop and Cheung [7] and calculated by an indirect procedure, requiring the knowledge of the complete wavefunction.

\section{The Calculation of the NO's}

The Hamiltonian $H$ of a two-electron system may be given in the usual from

$$
H=h(1)+h(2)+g(1,2)
$$

Reprint requests to Prof. Dr. H. Kleindienst, Institut für Physikalische Chemie I, Universität Düsseldorf, Universitätsstraße 1, D-4000 Düsseldorf. with the well known NO-expansion of the (singlet) ground-state eigenfunction $\psi$ as

$$
\Psi(1,2)=\sum_{i} c_{i} \chi_{i}(1) \chi_{i}(2), \quad\left(\chi_{i} \mid \chi_{j}\right)=\delta_{i j} .
$$

Independent variation [3] of the energy

$$
\begin{aligned}
& E=2 \sum_{i=1}^{n} c_{i}^{2} h_{i i}+\sum_{i, j}^{n} c_{i} c_{j} K_{i j}, \\
& h_{i i}=\left(\chi_{i} \mid h \chi_{i}\right), \quad K_{i j}=\left(\chi_{i} \chi_{i} \mid g \chi_{j} \chi_{j}\right)
\end{aligned}
$$

with respect to the $\chi_{i}=\chi_{i}^{(n)}$ and the $c_{i}=c_{i}^{(n)}$ limited to a $n$-dimensional subspace yields the system of integrodifferential equations

$$
\begin{aligned}
& c_{i}^{2} h \chi_{i}+\sum_{j=1}^{n} c_{i} c_{j} K_{j} \chi_{i}=\sum_{j=1}^{n} \lambda_{i j} \chi_{j}, \quad(i=1, \ldots, n), \\
& 2 c_{i} h_{i i}+\sum_{j=1}^{n} c_{j} K_{i j}=E c_{i}, \\
& \left(\chi_{i} \mid \chi_{j}\right)=\delta_{i j}, \quad(i, j=1, \ldots, n)
\end{aligned}
$$

with the usual definition of the exchange operator

$$
K_{j} \chi_{i}(1)=\left(\int \chi_{i}(2) g(1,2) \chi_{j}(2) \mathrm{d} \tau_{2}\right) \chi_{j}(1)
$$

and the Lagrange parameter $\lambda_{i j}=\lambda_{j i}$.

There are as many equations as unknowns, namely $2 n$ for the $\chi_{i}$ and $c_{i}$; for the $\frac{1}{2}\left(n^{2}+n\right)$ Lagrangeparameter $\lambda_{i j}$ there is exactly the same number of constraints - cf. (3) - from the orthonormality of the NO's $\chi_{i}$.

The solution of the system is obtained by a two step iteration procedure, decoupling the system partially. Equation (2) is used to determine $E$ and the $c_{i}$; the $\chi_{i}$ are obtained from (1) under the constraints (3). The method is demonstrated for the case $n=2$; an extension for $n>2$ is performed analogously.

0340-4811/ 85 / 1000-0995\$01.30/0. - Please order a reprint rather than making your own copy. 
Expansion and approximation of the $\chi_{i}$ with respect to the complete orthonormal system $\left\{\Phi_{k}\right\}$

$$
\chi_{i}=\sum_{k=1}^{m} a_{k}^{i} \Phi_{k}, \quad\left(\Phi_{j} \mid \Phi_{k}\right)=\delta_{j k}
$$

yields the matrixformulation of the system of integrodifferential equations above:

$$
\begin{array}{ll}
F^{1} \chi_{1}=i_{11} \chi_{1}+i_{12} \chi_{2}, & \left(\chi_{i} \mid \chi_{j}\right)=\delta_{i j}, \\
F^{2} \chi_{2}=\lambda_{12} \chi_{1}+i_{22} \chi_{2}, & (i, j=1,2), \\
A \boldsymbol{c}=E \boldsymbol{c} &
\end{array}
$$

with

$$
\begin{aligned}
F_{j k}^{i} & =\left(\Phi_{j} \mid F^{i} \Phi_{k}\right), \\
F^{i} & =c_{i}^{2} h+c_{i} c_{1} K_{1}+c_{i} c_{2} K_{2},(i=1,2), \\
A & =\left(\begin{array}{cc}
2 h_{11}+K_{11} & K_{12} \\
K_{12} & 2 h_{22}+K_{22}
\end{array}\right), \\
c & =\left(\begin{array}{l}
c_{1} \\
c_{2}
\end{array}\right) .
\end{aligned}
$$

With known values for the $c_{i}$ and $\chi_{i}$ from a former iteration cycle the matrix $A$ is estimated first, then $E$ and new $c_{i}$ are obtained from (5) by matrix diagonalization. With these new $c_{i}$ but the old $\chi_{i}$ the matrices $F^{1}$ and $F^{2}$ are constructed.

New $\chi_{i}$, i.e. new $a_{k}^{i}$, are obtained from the minimum of the quadratic form

$$
\begin{aligned}
& \begin{aligned}
F\left(\chi_{1}, \chi_{2}\right) & =\left(\chi_{1} \mid F^{1} \chi_{1}\right)+\left(\chi_{2} \mid F^{2} \chi_{2}\right) \\
& =\sum_{j, k=1}^{m}\left(F_{j k}^{1} a_{j}^{1} a_{k}^{1}+F_{j k}^{2} a_{j}^{2} a_{k}^{2}\right),
\end{aligned} \\
& g_{1}=\left(\chi_{1} \mid \chi_{1}\right)-1=0, \quad g_{3}=\left(\chi_{1} \mid \chi_{2}\right)=0 . \\
& g_{2}=\left(\chi_{2} \mid \chi_{2}\right)-1=0,
\end{aligned}
$$

This statement follows from the fact that the determination of the $\chi_{i}$ from (4) is equivalent to the calculation of the minimum of the quadratic functional (6) under the constraints (7) in a $2 m$-dimensional Hilbert space. The introduction of a $2 m$-dimensional space is necessary because there are $2 \mathrm{~m}$ independent variables $a_{i}^{1}$ and $a_{i}^{2}(i=1, \ldots, m)$ in the quadratic functional $F\left(\chi_{1}, \chi_{2}\right)$ of (6). The equivalence follows from the Euler-equations

$$
\nabla F+\mu_{1} \nabla g_{1}+\mu_{2} \nabla g_{2}+\mu_{3} \nabla g_{3}=0
$$

of the given variational problem: $\min F$ under $g_{k}=0$. Equation (8) written out in full is

$$
\begin{aligned}
& 2 F^{1} \chi_{1}+2 \mu_{1} \chi_{1}+\mu_{3} \chi_{2}=0, \\
& 2 F^{2} \chi_{2}+\mu_{3} \chi_{1}+2 \mu_{2} \chi_{2}=0 .
\end{aligned}
$$

The equivalence of (6)-(7) with (4)-(5) is given directly by (9) with

$$
\mu_{1}=-\lambda_{11}, \quad \mu_{2}=-\lambda_{22}, \quad \mu_{3}=-2 \lambda_{12} .
$$

For the calculation of the minimum of $F$ under the constraints $g_{k}=0$ we take advantage of the fact that the gradient $\nabla F$ is orthogonal to the tangent plane of the directions allowed by the constraints, i.e.

$$
\left(\nabla F \nabla g_{k}\right)=0, \quad(k=1,2,3) \text {. }
$$

The direction $z=\left(\begin{array}{l}z_{1} \\ z_{2}\end{array}\right)$ orthogonal to the gradients of the constraints is given by

$$
\begin{aligned}
z_{1}= & F^{1} \chi_{1}-\left(\chi_{1} F^{1} \chi_{1}\right) \chi_{1} \\
& -\frac{1}{2}\left[\left(\chi_{2} \mid F^{1} \chi_{1}\right)+\left(\chi_{1} \mid F^{2} \chi_{2}\right)\right] \chi_{2}, \\
z_{2}= & F^{2} \chi_{2}-\left(\chi_{2} \mid F^{2} \chi_{2}\right) \chi_{2} \\
& -\frac{1}{2}\left[\left(\chi_{2} F^{1} \mid \chi_{1}\right)+\left(\chi_{1} \mid F^{2} \chi_{2}\right)\right] \chi_{1} .
\end{aligned}
$$

This is easily verified by $\left(z \mid \nabla g_{k}\right)=0$.

With $y_{i}(t)=\chi_{i}(t)+t z_{i},(i=1,2), t \in \mathbb{R}$ the gradient $F\left(y_{1}(t), y_{2}(t)\right)$ is varied until $F$ is stationary, i.e. $t$ is determined from

$$
(\nabla F \boldsymbol{z})=0 .
$$

This yields

$$
t=-\frac{\left(z_{1} F^{1} \chi_{1}\right)+\left(z_{2} \mid F^{2} \chi_{2}\right)}{\left(z_{1} \mid F^{1} z_{1}\right)+\left(z_{2} \mid F^{2} z_{2}\right)} .
$$

The $y_{i}(t)$ obtained from stationary $F$ are orthonormalized and the whole process is performed until self consistence is reached.

For this usually good converging iteration process starting values of $c_{i}$ and $\chi_{i}$ are needed. Because the system of integrodifferential equations for $n=1,2, \ldots$ is solved successively we get a good approximation with $\chi_{1}=\chi_{\mathrm{HF}}$ for $n=1$. In the case $n=2$ we choose $c_{1}<1, c_{2}>0$ and $c_{1}^{2}+c_{2}^{2}=1$ without further restrictions. An approximation for $\chi_{2}$ is obtained from

$$
F^{2} \chi_{2}=i_{12} \chi_{1}+i_{22} \chi_{2}
$$

with $i_{12}=0$, which now is of the Hartree-Fock type. 
Table 1. Values of $E, F, \lambda_{i j}$ and $a_{k}^{i}$ for three NO's in a.u.

\begin{tabular}{lrrrrr}
\hline$E=-2.87886947$ & $F=-0.94597377$ \\
\cline { 3 - 5 }$c_{1}=0.99782$ & $c_{2}=-0.06555$ & \multicolumn{1}{l}{$c_{3}=-0.00812$} \\
\hline$\chi_{1}:$ & 0.93463 & -0.32385 & 0.13740 & -0.04861 & 0.01729 \\
& -0.00612 & 0.00199 & -0.00072 & 0.00022 & -0.00007 \\
$\chi_{2}:$ & 0.35429 & 0.81928 & -0.43565 & 0.10008 & -0.05606 \\
& 0.01618 & -0.00497 & 0.00330 & -0.00063 & 0.00079 \\
$\chi_{3}:$ & -0.02596 & -0.46433 & -0.85520 & 0.18170 & 0.05406 \\
& 0.12098 & 0.03037 & 0.02262 & -0.01811 & -0.00488 \\
$\lambda_{11}=-0.93306$ & $\lambda_{12}=-0.02126$ & $\lambda_{13}=0.00028$ \\
$\lambda_{22}=-0.012413$ & $\lambda_{23}=-0.00068$ & $\lambda_{33}=-0.00051$
\end{tabular}

\section{Discussion of the Result}

The method was tested for the He-atom in the radial-limit-approximation, with the basis $(m=10)$

[1] R. Ahlrichs, H. Lischka, and V. Staemmler, J. Chem. Phys. 62, 1225 (1975).

[2] P. O. Löwdin and H. Shull, Phys. Rev. 101, 1730 (1955).

[3] W. Kutzelnigg, Theor. Chim. Acta (Berl.) 1, 327 (1963).

[4] C. E. Reid and Y. Öhrn, Rev. Mod. Phys. 35, 445 (1963). and the integrals $(\eta=2.5)$ taken from Davis [8]. In addition to the values of the $c_{i}$ 's, $\chi_{i}^{\text {'s }}$ and $E$ for $n=3$ the minimal value of $F$ and the Lagrangeparameter $\lambda_{i j}$ with

$$
i_{i j}=\left(\chi_{i} \mid F^{j} \chi_{j}\right), \quad(i, j=1,2,3)
$$

are calculated.

The convergence of the gradient method is rapid, only a few (about 8 ) iterations are required. Convergence problems as mentioned e.g. in [5] do not occur.

\section{Acknowledgement}

We appreciate the assistence of Dr. G. Petermann, Institut für Physikalische Chemie I der Universität Düsseldorf, in translating this paper.

[5] R. Ahlrichs and F. Driessler, Theor. Chim. Acta (Berl.) 36, 275 (1975).

[6] E. R. Davidson, J. Chem. Phys. 37, 577 (1962).

[7] D. M. Bishop and L. M. Cheung, Int. J. Quant. Chem. 15, 517 (1979).

[8] H. L. Davis, J. Chem. Phys. 64, 2706 (1976). 